\title{
Gab family proteins are essential for postnatal maintenance of cardiac function via neuregulin-1/ErbB signaling
}

\author{
Yoshikazu Nakaoka, ${ }^{1}$ Keigo Nishida, ${ }^{2}$ Masahiro Narimatsu, ${ }^{3}$ Atsunori Kamiya, ${ }^{4}$ Takashi Minami,
} Hirofumi Sawa, ${ }^{6}$ Katsuya Okawa, ${ }^{7}$ Yasushi Fujio, ${ }^{8}$ Tatsuya Koyama, ${ }^{1}$ Makiko Maeda, ${ }^{8}$ Manami Sone, ${ }^{1}$ Satoru Yamasaki, ${ }^{2}$ Yuji Arai, ${ }^{9}$ Gou Young Koh, ${ }^{10}$ Tatsuhiko Kodama, ${ }^{5}$ Hisao Hirota, ${ }^{11}$ Kinya Otsu, ${ }^{11}$ Toshio Hirano, ${ }^{2,3}$ and Naoki Mochizuki ${ }^{1}$

${ }^{1}$ Department of Structural Analysis, National Cardiovascular Center Research Institute, Osaka, Japan. 2Laboratory for Cytokine Signaling, RIKEN Research Center for Allergy and Immunology (RCAI), Yokohama, Japan. ${ }^{2}$ Laboratory of Developmental Immunology,

Osaka University Graduate School of Frontier Biosciences and Graduate School of Medicine, Osaka, Japan. ${ }^{4}$ Department of Cardiovascular Dynamics, National Cardiovascular Center Research Institute, Osaka, Japan. ${ }^{5}$ Laboratory for System Biology and Medicine,

Research Center for Advanced Science and Technology, University of Tokyo, Tokyo, Japan. ${ }^{6}$ Department of Molecular Pathobiology, Hokkaido University Research Center for Zoonosis Control, Sapporo, Japan. ${ }^{7 H}$ orizontal Medical Research Organization,

Kyoto University Graduate School of Medicine, Kyoto, Japan. ${ }^{8}$ Department of Clinical Evaluation of Medicines and Therapeutics, Osaka University Graduate School of Pharmaceutical Sciences, Osaka, Japan. 9Department of Bioscience, National Cardiovascular Center Research Institute, Osaka, Japan. ${ }^{10 B i o m e d i c a l ~ R e s e a r c h ~ C e n t e r ~ a n d ~ D e p a r t m e n t ~ o f ~ B i o l o g i c a l ~ S c i e n c e s, ~ K o r e a ~ A d v a n c e d ~ I n s t i t u t e ~ o f ~ S c i e n c e ~ a n d ~ T e c h n o l o g y, ~ D a e j e o n, ~}$ Republic of Korea. ${ }^{11}$ Department of Cardiovascular Medicine, Osaka University Graduate School of Medicine, Osaka, Japan.

Grb2-associated binder (Gab) family of scaffolding adaptor proteins coordinate signaling cascades downstream of growth factor and cytokine receptors. In the heart, among EGF family members, neuregulin-1 $\beta$ (NRG-1 $\beta$, a paracrine factor produced from endothelium) induced remarkable tyrosine phosphorylation of Gab1 and Gab2 via erythroblastic leukemia viral oncogene (ErbB) receptors. We examined the role of Gab family proteins in NRG-1 $\beta$ /ErbB-mediated signal in the heart by creating cardiomyocyte-specific Gab1/Gab2 double knockout mice (DKO mice). Although DKO mice were viable, they exhibited marked ventricular dilatation and reduced contractility with aging. DKO mice showed high mortality after birth because of heart failure. In addition, we noticed remarkable endocardial fibroelastosis and increase of abnormally dilated vessels in the ventricles of DKO mice. NRG-1 $\beta$ induced activation of both ERK and AKT in the hearts of control mice but not in those of DKO mice. Using DNA microarray analysis, we found that stimulation with NRG-1 $\beta$ upregulated expression of an endothelium-stabilizing factor, angiopoietin 1, in the hearts of control mice but not in those of DKO mice, which accounted for the pathological abnormalities in the DKO hearts. Taken together, our observations indicated that in the NRG-1 $\beta /$ ErbB signaling, Gab1 and Gab2 of the myocardium are essential for both maintenance of myocardial function and stabilization of cardiac capillary and endocardial endothelium in the postnatal heart.

\section{Introduction}

Dilated cardiomyopathy (DCM) is an common cause of heart failure. Epidemiological studies suggest that $25 \%-30 \%$ of DCM is inherited. Among the mutations associated with DCM in humans and mice, several involve genes encoding cytoskeletal proteins and sarcomere-related proteins (1); however, mutations in these known genes account for only a minor proportion of the heritable cardiomyopathies in humans. Cardiac function is maintained by cytokine- and growth factor-triggered intracellular signaling. Genetically modified mice, in which intracellular signaling molecules are either activated or perturbed, also exhibit cardiac

Nonstandard abbreviations used: Ang1, angiopoietin 1; ANP, atrial natriuretic peptide; DCM, dilated cardiomyopathy; DKO, cardiomyocyte-specific Gab1/Gab2 double knockout; EFE, endocardial fibroelastosis; EphA4, Eph receptor A4; ErbB, erythroblastic leukemia viral oncogene; Gab, Grb2-associated binder; Gab1CKO, cardiomyocyte-specific Gab1 conditional knockout; Gab1KO, conventional Gab1 knockout; HB-EGF, heparin-binding EGF-like growth factor; LIF, leukemia inhibitory factor; $\alpha$-MHC, $\alpha$-myosin heavy chain; NRG-1, neuregulin- $1 ; \alpha$-SKA, skeletal $\alpha$-actin; TSP1, thrombospondin 1 .

Conflict of interest: The authors have declared that no conflict of interest exists. Citation for this article: J. Clin. Invest. 117:1771-1781 (2007). doi:10.1172/JCI30651. dysfunction, suggesting that coordination of signal transduction systems is critical for the preservation of cardiac function (2).

The Grb2-associated binder (Gab) family proteins, which serve as scaffolding adaptor proteins, crucially intervene between receptors and intracellular signaling molecules to coordinate the signaling cascades of cytokines, growth factors, antigens, and numerous other molecules (3-5). Multiple phosphorylated tyrosine residues of Gab proteins become docking sites for Src homology-2 domaincontaining molecules. Docking of Gab to tyrosine phosphatase SHP2 and the $\mathrm{p} 85$ regulatory subunit of PI3K leads to the activation of ERK and AKT, respectively $(4,5)$. Three Gab family members, Gab1, Gab2, and Gab3, have been identified in mammals and are structurally similar $(4,5)$. Conventional Gab1 knockout (Gab1KO) mice display embryonic lethality with impaired development of heart, placenta, skin, and muscle $(6,7)$. Gab2KO mice do not show any obvious developmental defects but display impaired allergic responses and osteoclast defects $(8-11)$. Gab3KO mice exhibit no obvious phenotype (12).

We previously demonstrated the importance of Gab1-ERK5 signaling in cardiomyocyte hypertrophy through the leukemia inhibitory factor-gp130-dependent (LIF-gp130-dependent) 

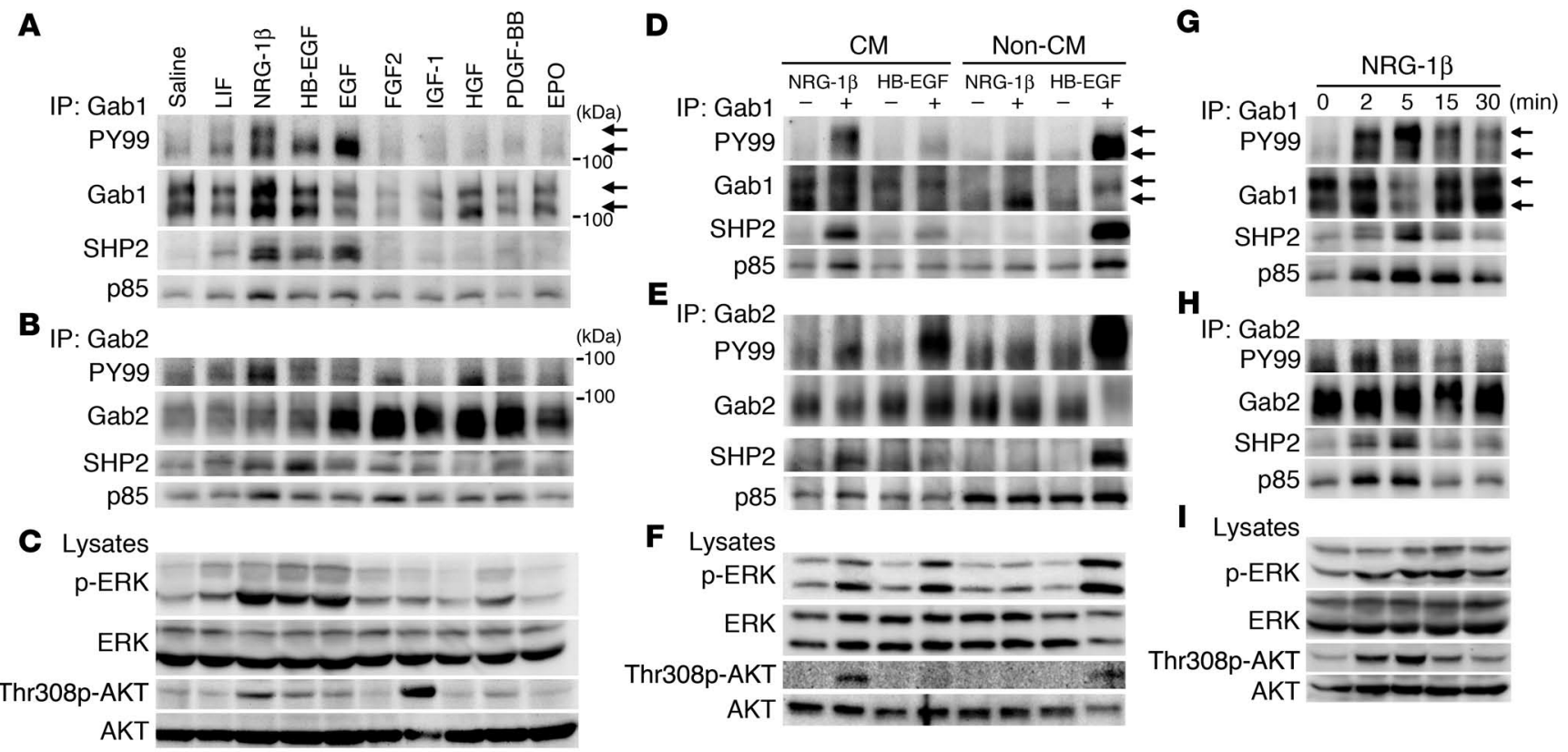

\section{Figure 1}

Gab1 and Gab2 are engaged in coordination of NRG-1 $\beta$ /ErbB signaling pathway in the myocardium. Tyrosine phosphorylation of Gab1 (A) and Gab2 (B) and their association with SHP2 and p85 were analyzed by IP of the heart lysates. Mouse heart lysates were prepared at 5 minutes after injection with the cytokines and growth factors listed at top. Heart lysates were subjected to IP with anti-Gab1 (A) or anti-Gab2 (B) serum, followed by IB analysis using the Ab indicated at the left. (C) Activation levels of ERK and AKT were assessed by phospho-specific Ab. Tyrosine phosphorylation of Gab1 (D) Gab2 (E) and their association with SHP2 and p85 was examined by IP of cell lysates from neonatal rat cardiomyocytes (CM) or noncardiomyocytes (non-CM) stimulated with either NRG-1 $\beta$ (50 ng/ml) or HB-EGF $(50 \mathrm{ng} / \mathrm{ml})$ for $5 \mathrm{minutes}$. IP complexes were subjected to IB using the Ab indicated at the left. (F) NRG-1 $\beta$ - and HB-EGF-dependent activation of ERK and AKT was examined in CM and non-CM as in $\mathbf{C}$. Tyrosine phosphorylation of Gab1 $(\mathbf{G})$ and $G a b 2(\mathbf{H})$ and their association with SHP2 and p85 in the mouse hearts were analyzed after injection with $5 \mu \mathrm{g}$ of NRG-1 $\beta$ as in $\mathbf{A}$ and $\mathbf{B}$, respectively. Heart lysates were prepared at the indicated time after injection. Gab1 and Gab2 underwent tyrosine phosphorylation and associated with SHP2 and p85 in a time-dependent manner upon NRG-1 $\beta$ stimulation. (I) Activation of ERK and AKT were assessed as in C. Arrows denote 2 isoforms of Gab1. Representative blots of 3 experiments are shown. PY99, antibody recognizing phospho-tyrosine.

signaling pathway (13). Gab family proteins are also involved in EGF family-erythroblastic leukemia viral oncogene (EGF family-ErbB) receptor family signaling $(6,14,15)$. EGF family-ErbB receptor signaling plays crucial roles in heart development and preservation of adult cardiac function $(16,17)$. Among the EGF family members, neuregulin-1 (NRG-1) (18) and heparin-binding EGF-like growth factor (HB-EGF) (19) are particularly important agonists for ErbB receptors on cardiomyocytes. NRG-1 serves as a paracrine factor that is shed from the endothelium and activates the ErbB4 homodimer or ErbB2/ErbB4 (also known as HER2/ HER4) heterodimer on cardiomyocytes (16, 17, 20, 21). NRG-1-, ErbB2-, and ErbB4-deficient mice display embryonic lethality and similar defects in ventricular trabeculation (22-24). HB-EGFdeficient mice also display abnormal valvular development and cardiac dysfunction $(25,26)$.

The importance of ErbB signaling in the adult heart was first revealed by the unforeseen adverse effects of trastuzumab (Herceptin), a monoclonal $\mathrm{Ab}$ against ErbB2 used in the treatment of breast cancer. Trastuzumab induces heart failure when combined with anthracycline treatment $(17,27,28)$. In addition to this clinical evidence, cardiomyocyte-specific ErbB2- and ErbB4deficient mice both exhibit DCM (29-31). However, the precise intracellular signaling responsible for ErbB-regulated cardiac function is still unclear.
In the present study, we used myocardium-specific deletion of Gab family proteins in the mice to demonstrate that Gab1 and Gab2 in the myocardium are essential for transmitting the signal from NRG-1 $\beta /$ ErbB to directly maintain myocardial function and to subsequently stabilize capillary and endocardial endothelium in the postnatal heart.

\section{Results}

Gab1 and Gab2 are engaged in coordination of $N R G-1 \beta / E r b B$ signaling pathway in the myocardium. We aimed at exploring the function of Gab family proteins in the heart. Thus, we first examined the expression of Gab family transcripts by RT-PCR and detected the mRNA of Gab1 and Gab2, but not that of Gab3, in the murine heart (Supplemental Figure 1; supplemental material available online with this article; doi:10.1172/JCI30651DS1). To elucidate how Gab1 and Gab2 are involved in the intracellular signaling in the heart, mice were injected with various cytokines and growth factors. Among these agonists, ErbB receptor-activating agonists, including NRG-1 $\beta$, HB-EGF, and EGF, induced strong tyrosine phosphorylation of Gab1 and Gab2 and the subsequent association of Gab1 and Gab2 with SHP2 and p85 (Figure 1, A and B). We identified 2 Gab1 isoforms, high-molecular weight (highMW) Gab1 (120-130 kDa) and low-MW Gab1 (100 kDa). Notably, the high-MW Gab1 underwent tyrosine phosphorylation 


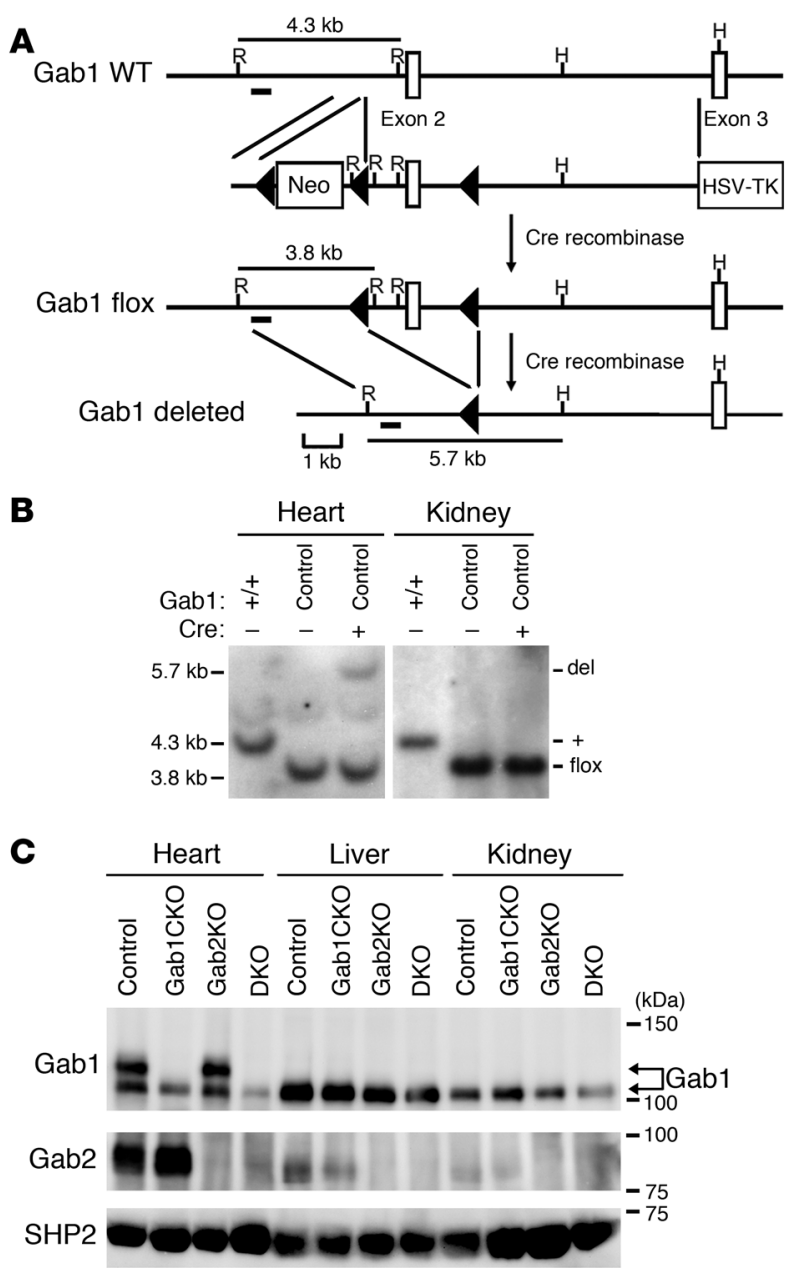

upon stimulation exclusively with NRG-1 $\beta$, while low-MW Gab1 was phosphorylated by NRG-1 $\beta$, HB-EGF, and EGF (Figure 1A). We confirmed that the high-MW Gab1 is a cardiac-specific isoform using molecular mass spectrometric analysis, which showed that the high-MW band that was recognized by anti-Gab1 Ab in Western blot analysis indeed contained the partial amino acid sequence of Gab1 (Supplemental Figure 2, A-C). Activation of both ERK and AKT was found only when stimulated with NRG-1 $\beta$, HB-EGF, and EGF (Figure 1C), although activation of AKT was most strongly induced by IGF-1.

We examined whether the difference in Gab1 phosphorylation was due to the diversity of the cell types. To distinguish the signaling processes in cardiomyocytes from those in noncardiomyocytes, including fibroblasts, endothelial cells, and vascular smooth muscle cells in the heart, we analyzed the action of NRG-1 $\beta$ and HB-EGF in neonatal rat cardiomyocytes and noncardiomyocytes that had been isolated using the Percoll gradient method (32). NRG-1 $\beta$ induced tyrosine phosphorylation of Gab1 and Gab2, the subsequent association of Gab1 and Gab2 with SHP2 and $\mathrm{p} 85$, and the activation of ERK and AKT in cardiomyocytes but not in noncardiomyocytes (Figure 1, D-F). In clear contrast, HB-EGF induced those changes more strongly in noncardiomyocytes than in cardiomyocytes (Figure 1, D-F). It should be noted that tyrosine phosphorylation of the high-MW Gab1 in cardiomyocytes was induced after stimulation with NRG-1 $\beta$ but not with

\section{Figure 2}

Generation of DKO mice. (A) Schematic illustration of genomic structure of the Gab1 wild-type, Gab1 flox, and Gab1-deleted alleles and a targeting vector. loxP sequences are indicated by black triangles. Restriction enzyme sites for EcoRI and HindIII are indicated as R and $\mathrm{H}$, respectively. Fragments detected by the probe (short bold line) used for Southern blot analysis after digestion of genomic DNA with EcoRI and HindIII are indicated as solid lines measuring $4.3 \mathrm{~kb}, 3.8 \mathrm{~kb}$, and $5.7 \mathrm{~kb}$. HSV-TK, herpes simplex virus-thymidine kinase. (B) Southern blot analysis demonstrated recombination of the Gab1tlox allele in the heart, but not in the kidney, of Gab1 floxflox mice, which possessed the $\alpha-M H C-C r e$ allele. (C) Following IP, expression of Gab1 and Gab2 was examined by IB using anti-Gab1 (top row) and anti-Gab2 (middle row) serums. SHP2 was examined as a loading control (bottom row). Note that 2 isoforms of Gab1 were detected at the different MW exclusively in the heart (arrows) and that the high-MW Gab1 isoform in the heart was completely depleted in Gab1CKO and DKO. The low-MW Gab1 was also reduced by $80 \%$ in the heart of Gab1CKO and DKO mice compared with control and Gab2KO mice.

HB-EGF (Figure 1D). These findings suggest that NRG-1 $\beta$ acts as a highly selective agonist for cardiomyocytes, in agreement with previous reports (33).

Therefore, we focused on the NRG-1 $\beta$-dependent signaling pathway through Gab1 and Gab2 in the murine hearts. Gab1 and Gab2 underwent tyrosine phosphorylation and associated with SHP2 and p85 after injection with NRG-1 $\beta$ in a time-dependent manner (Figure 1, $\mathrm{G}$ and $\mathrm{H}$ ). In addition, both ERK and AKT were also activated by NRG-1 $\beta$ in a time-dependent manner (Figure 1I). We also checked the activation of ErbB family receptors of murine hearts stimulated with NRG-1 $\beta$. NRG- $1 \beta$ induced tyrosine-phosphorylation of ErbB2 and ErbB4 but not that of ErbB1 (EGFR) or ErbB3 in accordance with a previous report in which cardiomyocytes were used in vitro (Supplemental Figure 3, A-D) (21). Furthermore, Gab1 associated with ErbB4 in a phosphorylationdependent manner after injection with NRG-1 $\beta$ (Supplemental Figure $3 \mathrm{E}$ ). These data suggest the engagement of Gab family proteins in the coordination of NRG-1 $\beta /$ ErbB signaling pathway.

Generation of cardiomyocyte-specific Gab1 conditional knockout mice. To elucidate the function of Gab family proteins in myocardium, we first generated cardiomyocyte-specific Gab1 conditional knockout (Gab1CKO) mice using the Cre-loxP system. Using homologous recombination in embryonic stem cells, we created a Gab1 flox allele by introducing 2 loxP sites into introns flanking exon 2, which encodes part of the pleckstrin homology domain 


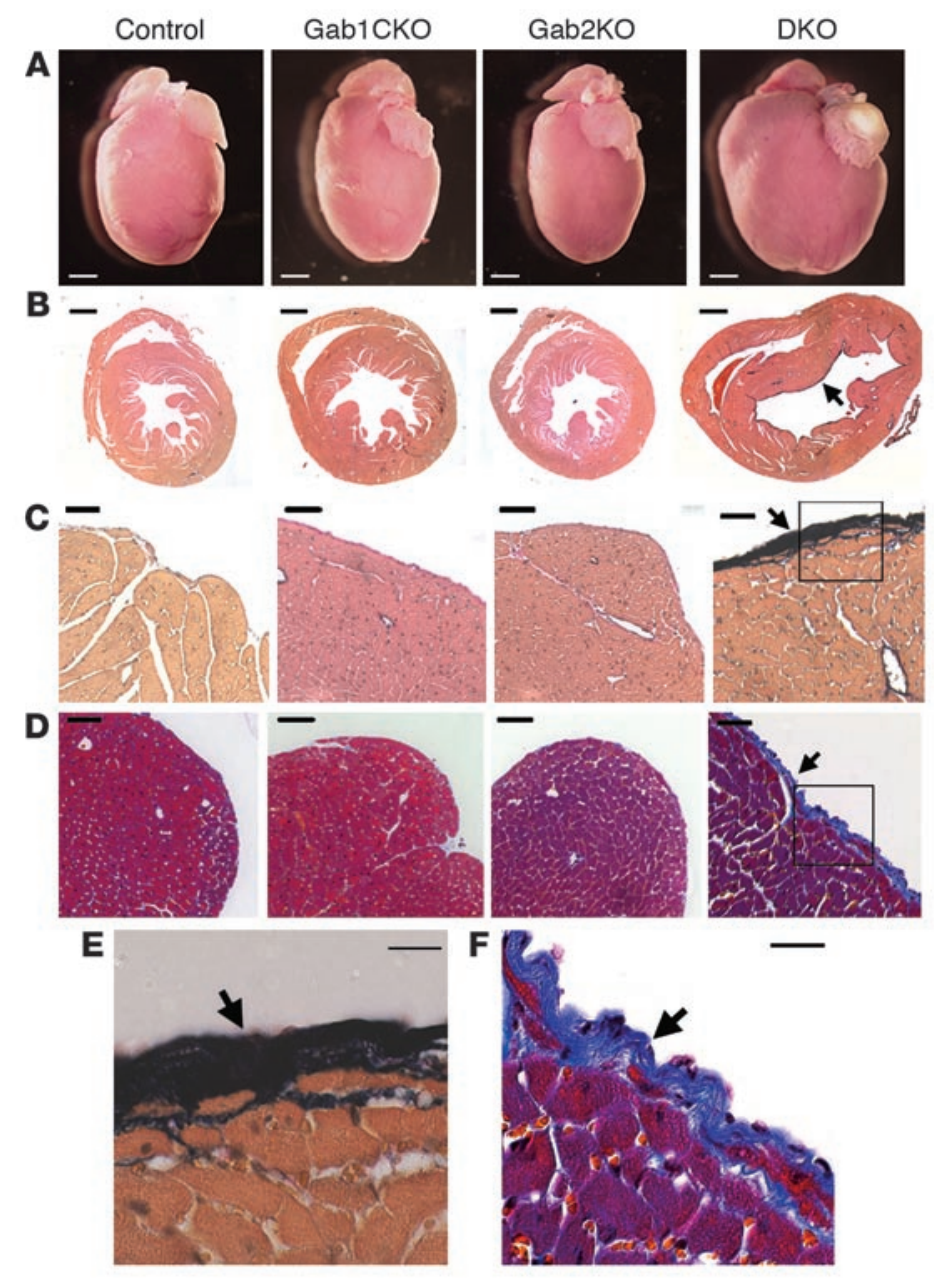

(Figure 2A). The protein expression of Gab1 in all tissues of mice homozygous for the Gab1-loxP-targeted allele (Gab1 $1^{\text {flox/flox }}$ mice) was almost the same level as in wild-type mice (data not shown). To cause recombination of the floxed allele exclusively in cardiomyocyte lineage, Gab1 floxfllox mice were crossed with transgenic mice expressing $\alpha$-myosin heavy chain promoter-driven Cre recombinase ( $\alpha$-MHC-Cre mice) $(34,35)$ (Figure 2A). We confirmed the Cremediated recombination during embryogenesis (E10.5 and E14.5) by crossing $\alpha-M H C-C r e$ mice with enhanced GFP reporter mice (Supplemental Figure 4A). The Gab1CKO (Gab1flox/flox $\alpha-M H C$ $\mathrm{Cre}(+))$ mice were born normally at the expected Mendelian frequency, whereas Gab1KO mice were embryonically lethal (6). In addition, the Gab1CKO mice displayed normal appearance and normal cardiac morphology at birth (Supplemental Figure 5A).

We observed the expected genetic recombination at the Gab1 locus in the ventricles of Gab1CKO mouse hearts but not in other tissues (Figure 2B). In order to estimate the expression of Gab1 protein, immunoblot analyses were performed using the extracts from heart, liver, and kidney (Figure 2C). As described above, 2 isoforms of Gab1 proteins were detected in hearts, while low-MW Gab1 was commonly detected, suggesting that the high-MW Gab1 is a cardiac-specific isoform. Moreover, high-MW Gab1 protein was deleted in Gab1CKO hearts, suggesting that high-MW Gab1 is a product of the same Gab1 gene that has low MW. In addition, we used Percoll gradient centrifugation to analyze the expression of Gab1 in car-

\section{Figure 3}

DKO mice display dilated cardiomyopathic features accompanied by EFE. (A) Representative images of whole hearts from 4 groups at 10 weeks of age. (B) Transverse sections of the hearts were stained using the elastica van Gieson method. DKO hearts showed marked biventricular dilation and slight wall thinning compared with the other 3 groups of hearts. (C and $\mathbf{E}$ ) Higher magnification of elastica van Gieson-stained section of DKO heart shows the focal accumulation of elastic fibers (black) in the endocardium (arrows in B and C). (D) Masson's trichromestained section of DKO heart shows focal accumulation of collagen (blue) in the endocardium (arrow in $\mathbf{D})$. (E and F) Boxed regions of $\mathbf{C}$ and $\mathbf{D}$, respectively, are enlarged. Scale bars: $1 \mathrm{~mm}$ (A and B); $20 \mu \mathrm{m}(\mathbf{C}-\mathbf{F})$.

diomyocytes and noncardiomyocytes isolated from neonatal rat hearts (32) and detected the high-MW isoform of Gab1 exclusively in cardiomyocytes (Supplemental Figure 2D).

In Gab1CKO mice, the high-MW Gab1 was completely deleted and the low-MW Gab1 was reduced to about $20 \%$ of control (Gab1 $1^{\text {flox/flox }}$ ) littermates. The residual low-MW Gab1 protein might be attributed to the noncardiomyocytes present in the heart. These data indicated the successful depletion of Gab1 in the cardiomyocytes (Figure 2C), because $\alpha-M H C$ promoter functions exclusively in the myocardium. In 3-dayold Gab1CKO mouse hearts, we detected an extent of Gab1 protein depletion similar to that of 3- or 10-week-old mice (Supplemental Figure 4B).

Generation of cardiomyocyte-specific Gab1/Gab2 double knockout mice. In murine hearts, mRNAs of Gab1 and Gab2 were detected by RT-PCR (Supplemental Figure 1). Gab2 can rescue the loss of Gab1 for activation of ERK in the EGF signaling pathway (36). We thus assumed that Gab2 might compensate for the deletion of Gab1 in the cardiomyocytes of Gab1CKO mice.

To completely deplete Gab family proteins in cardiomyocytes, Gab1CKO mice were crossed with Gab2KO mice. We created

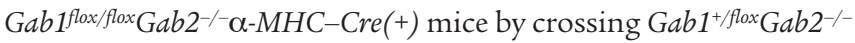
$\alpha-M H C-C r e(+)$ mice with Gab1flox/flox $G a b 2^{-1-\alpha-M H C-C r e(-) ~ m i c e ~ i n ~}$ the final breeding. The offspring of these crossings were recovered at expected Mendelian ratios as follows: $\mathrm{Gab1}^{+/ f l o x} \mathrm{Gab2^{-/- } \alpha - M H C -}$ $\mathrm{Cre}(-)$ ( $n=44 ; 24.6 \%) ; G a b 1^{+/ f l o x} \mathrm{Gab2}^{-/-} \alpha-\mathrm{MHC}-\mathrm{Cre}(+)$ ( $n=46$; 25.7\%); Gab1 flox/flox $\mathrm{Gab2} 2^{-/-} \alpha-M H C-C r e(-)$ ( $\left.n=39 ; 21.8 \%\right) ; G a b 1^{\text {flox/flox }}$ Gab2 ${ }^{-1-\alpha-M H C-C r e}(+)(n=50 ; 27.9 \%)$. Thereafter, we analyzed the following 4 groups of mice: Gab1 $1^{\text {flox/flox }} \mathrm{Gab2}{ }^{+/+} \alpha-M H C-C r e(-)$

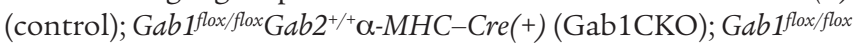
Gab2 ${ }^{-/-} \alpha-M H C-C r e(-)(G a b 2 \mathrm{KO})$; and Gab1 $1^{\text {flox/flox }} \mathrm{Gab2} 2^{-/-} \alpha-M H C-$ Cre(+) (DKO). Both Gab2KO and DKO mice displayed normal appearance and normal cardiac morphology at birth (Supplemental Figure 5A). Gab2 protein was completely depleted in the Gab2KO and DKO mice, indicating the successful depletion of Gab1 and Gab2 in the cardiomyocytes of DKO mice (Figure 2C).

DKO mice display dilated cardiomyopathic features accompanied by endocardial fibroelastosis. We performed gross morphological examination of the hearts of the 4 groups at 10 weeks of age because we did not find any morphological abnormalities in the hearts of Gab1CKO, Gab2KO, or DKO mice at birth (Supplemental Figure $5 \mathrm{~A})$. Although there was no morphological difference among Gab1CKO, Gab2KO, and control mice (Figure 3A), DKO mice exhibited significantly higher heart weight-to-body weight ratios 
A

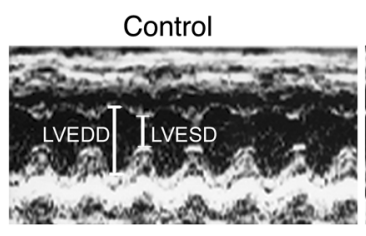

Gab1CKO

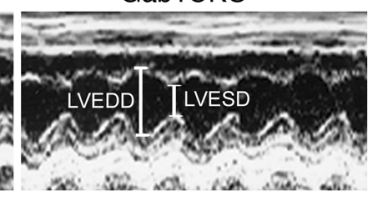

Gab2KO

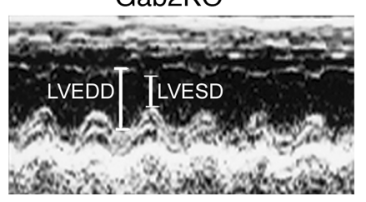

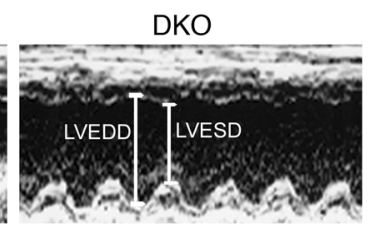
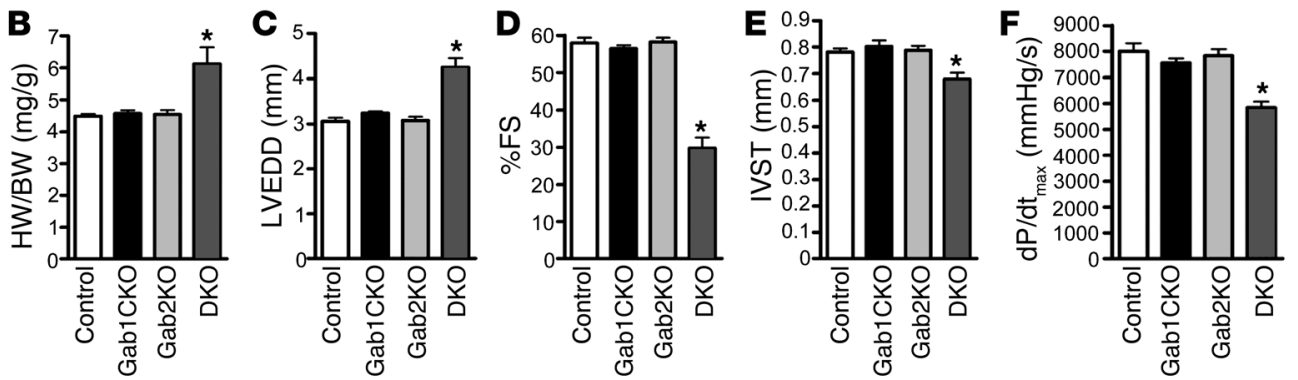

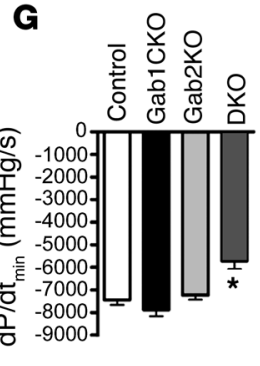

Figure 4

DKO mice exhibit dilated cardiomyopathic features. (A) Representative examples of M-mode echocardiographic images of LV from each group of mice at 10 weeks of age. LVEDD, LV end-diastolic dimension; LVESD, LV end-systolic dimension. (B) Heart weight/body weight (HW/BW) ratio of control mice $(n=9)$, Gab1CKO mice $(n=6)$, Gab2KO mice $(n=6)$, and DKO mice $(n=10)$ at 10 weeks of age. (C) LVEDD, (D) fractional shortening (\%FS), and (E) interventricular septal thickness (IVST) of control mice $(n=8)$, Gab1CKO mice $(n=8)$, Gab2KO mice $(n=7)$, and DKO mice $(n=14)$ at 10 weeks of age. There were no significant differences in BW or heart rate among the 4 groups. (F) The maximum

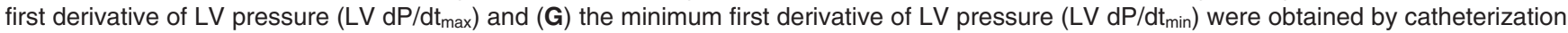
of LV from right carotid artery in control mice $(n=7)$, Gab1CKO mice $(n=6)$, Gab2KO mice $(n=7)$, and DKO mice $(n=7)$ at 12 weeks of age. ${ }^{\star} P<0.01$ compared with all other genotypes.

than the other 3 groups without significant differences in body weight (Figure $3 \mathrm{~A}$ and Figure 4B). Histological examination also demonstrated both left and right ventricular enlargement in DKO mice similar to DCM (Figure 3B).

A significant accumulation of elastic fibers and collagen was observed exclusively in the endocardium of DKO mice (Figure 3, B-F), while fibrotic replacement was not found in the interstitial spaces of the ventricles of DKO mice (Supplemental Figure 6, A and B). There was no significant increase in the number of apoptotic myocardial cells in the hearts of DKO mice compared with those of control mice (Supplemental Figure 7, A and B). The endocardial deposition of elastic fibers and collagen was not found in the neonates of DKO, but was found to some extent in all of the DKO mice after 3 weeks (Supplemental Figure 5A and data not shown). These endocardium-specific changes were coincident with the pathological features of endocardial fibroelastosis (EFE), the genetic causality of which has not been fully elucidated to date $(37,38)$. We further examined the vasculature in the heart by immunostaining with antivWF Ab. Intriguingly, we found abnormally dilated vessels positively stained with anti-vWF Ab exclusively in the LV of DKO mice but not in those of control, Gab1CKO, or Gab2KO mice (Figure 5A). These dilated vessels in DKO mice exhibited the impairment in recruitment of $\alpha$-SMA-positive VSMCs (Figure 5, B and C). These findings indicate that the maintenance system for both endocardial and vascular endothelium might be disturbed in the DKO mouse hearts. Furthermore, EFE and increased abnormal vessels in the hearts of DKO mice were indirectly ascribed to the lack of Gab1 and Gab2 in the myocardium because there was no abnormality in the other 3 groups.

We assessed in vivo cardiac function by echocardiography and cardiac catheterization. Echocardiography revealed a significant increase in LV end-diastolic dimension (Figure 4, A and C), decreased fractional shortening (Figure 4, A and D), and decreased interventricular septal wall thickness (Figure 4E) in 10-week-old
DKO mice compared with age-matched mice of the other 3 groups. Although we did not find a significant changes of LV end-diastolic dimension or fractional shortening between the DKO and control mice at 3 weeks of age, we did observe these changes after 6 weeks of age (Supplemental Figure 8, A and B). Consistent with the echocardiographic findings, cardiac catheterization at 12 weeks of age revealed a marked reduction of the maximum first derivative of $\mathrm{LV}$ pressure exclusively in DKO (Figure 4F), demonstrating a reduction in myocardial contractility of the DKO hearts. The accompanying reduction of the minimum first derivative of $L V$ pressure in the DKO mouse hearts indicated the impairment of LV relaxation (Figure 4G). There were no significant differences in heart rate or LV peak pressure among the 4 groups (data not shown). This relaxation failure was supported by the electron microscopic findings. We noticed that sarcomere length was reduced in the DKO mouse hearts, which indicated the hypercontraction phenotype (39), although we could detect slight changes in the mitochondria of DKO mouse hearts (Supplemental Figure 7, C and D). In agreement with the reduced contractility and relaxation reflecting heart failure, the fetal cardiac gene program was reactivated, as evidenced by the significant increase in both atrial natriuretic peptide (ANP) and skeletal $\alpha$-actin ( $\alpha-S K A)$ mRNAs in DKO mice (Figure 6, A-C).

Approximately $70 \%$ of the DKO mice died, presumably of heart failure accompanied by pleural effusion, between 3 and 72 weeks of age (Figure 6D). We observed remarkably dilated ventricles in DKO mice that had died of heart failure (Supplemental Figure $5 \mathrm{~B}$, right panel). The other 3 groups of mice lived normally during the observation period of 500 days (Figure 6D). In agreement with this survival analysis, we did not observe any enlargement of the hearts of Gab1CKO and Gab2KO mice at 300 and 500 days of age. (Supplemental Figure 5B and data not shown). These data indicate that depletion of both Gab1 and Gab2 in the myocardium result in DCM-like phenotype accompanied by EFE. 
A Control

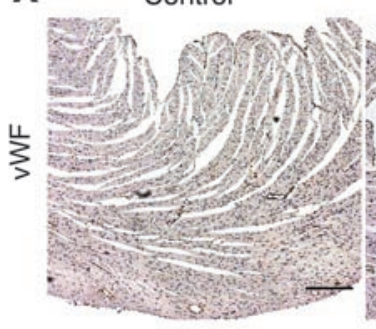

B

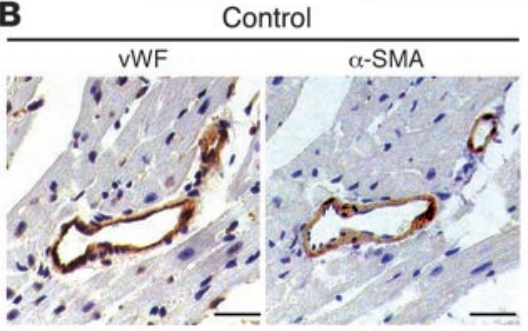

Gab1CKO

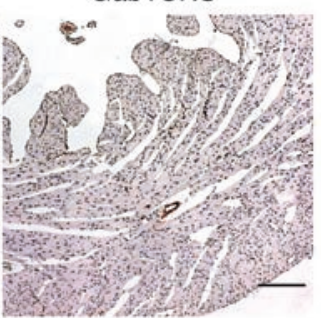

C
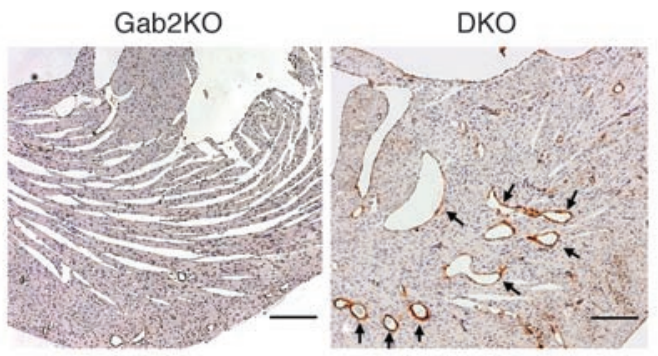

DKO

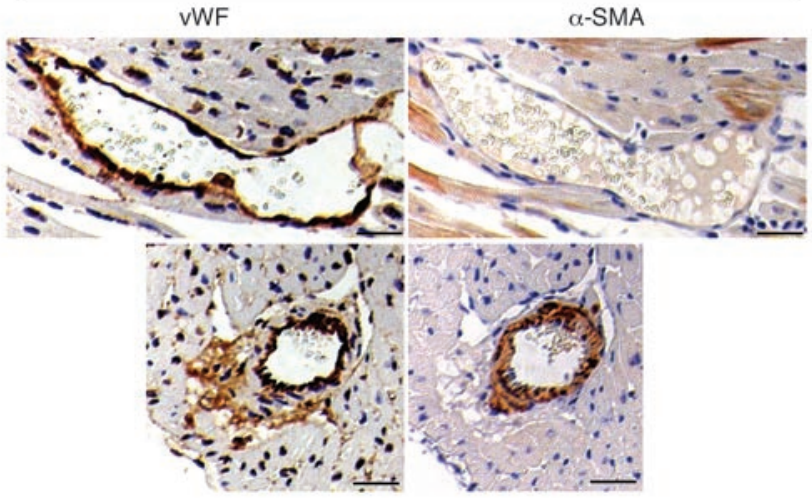

Figure 5

DKO mice display vascular abnormalities in the ventricles. (A) Heart sections from 4 groups of mice at 6 weeks of age were immunostained with antivWF Ab. vWF-positive, abnormally dilated vessels were observed in the left ventricles of DKO mice (arrows) but not in those of control, Gab1CKO, or Gab2KO mice. Representative photographs are shown. (B and C) Heart sections from control (B) and DKO (C) mice at 6 weeks of age were immunostained with anti-vWF and anti- $\alpha$-SMA Abs. The abnormally dilated vessels in DKO mice were not surrounded by $\alpha$-SMA-positive VSMCs in most cases (C, top panels), although vessels of normal diameter near the epicardium in DKO mice were surrounded by $\alpha$-SMA-positive VSMCs (C, bottom panels) as observed in control mice (B). Representative images are shown. Scale bars: $200 \mu \mathrm{m}(\mathbf{A}) ; 20 \mu \mathrm{m}(\mathbf{B}$ and $\mathbf{C})$.

Gab1 and Gab2 are required for NRG-1 $\beta /$ ErbB signaling in the heart. To determine requirements of Gab1 and Gab2 in NRG-1 $\beta$-triggered signaling in the myocardium, we examined the activation of ERK and AKT after injection of NRG-1 $\beta$. NRG- $1 \beta$-induced activation of ERK and AKT was completely abrogated in DKO mice but not in the other 3 groups (Figure 7, A-C), suggesting a compensatory function of Gab1 and Gab2 in the heart. Consistently, tyrosine phosphorylation of Gab1 and subsequent association with SHP2 and p85 were observed in control and Gab2KO mice but not in Gab1CKO or DKO mice (Figure 7D). Tyrosine phosphorylation of Gab2 and subsequent association with SHP2 and p 85 were conversely observed in control or Gab1CKO mice but not in Gab2KO or DKO mice (Figure 7E). Tyrosine phosphorylation of ErbB2 and ErbB4 was comparable among the 4 groups (Figure 7F). IGF-1- and HB-EGF-dependent activation of ERK and AKT were not affected in the hearts of DKO mice (Supplemental Figure 9, A and B). These data indicate that Gab1 and Gab2 are required exclusively for NRG-1 $\beta /$ ErbB signaldependent activation of ERK and AKT in the heart.

Angiopoietin 1 upregulation induced by NRG-1 $\beta$ is impaired in Gab1/ Gab2-deficient myocardium. Because we observed no cardiac abnormalities in Gab2KO mice, we determined that the primary cause of EFE and abnormal vessels in DKO mouse hearts was not the lack of Gab2 in endothelial cells. To identify the potential signal defect that caused EFE and malformed vessels downstream of the NRG-1 $\beta /$ ErbB-Gab1/Gab2 signaling pathway in the myocardium, we used microarrays to carry out a global survey of mRNA in control and DKO mice treated with or without NRG-1 $\beta$ for 8 hours.
We found several transcripts that were upregulated by stimulation with NRG-1 $\beta$ in the hearts of control mice but not in those of DKO mice (Figure 8A). Among these transcripts presented in the cluster diagram, we considered thrombospondin 1 (TSP1) and angiopoietin 1 (Ang1) to be potential paracrine factors from myocardium and Eph receptor A4 (EphA4) to be important for the intercellular communication between cardiomyocytes and surrounding cells.

To address the pathogenesis of endocardial and vascular abnormalities observed in DKO mouse hearts, we focused on Ang1 because it has an important role in maturation of both vascular endothelium and endocardial endothelium in vivo (40-42). We confirmed by northern blot analysis that NRG-1 $\beta$ upregulated Ang1 mRNA in the hearts of control mice, but not DKO mice (Figure 8, B and C). NRG-1 $\beta$ consistently induced significant upregulation of Ang1 mRNA in cultured cardiomyocytes but not in noncardiomyocytes (Figure 8, D-F). In association with defective expression of Ang1, CD31-positive capillary density was significantly decreased in the LV of DKO mice compared with control (Figure 8, G and $\mathrm{H}$ ). Taken together, these findings suggest that the lack of NRG-1 $\beta$-induced upregulation of Ang 1 might be one of the possible causes for pathogenesis of EFE and abnormal vasculatures in DKO mouse hearts.

\section{Discussion}

To our knowledge, the present study is the first to reveal the

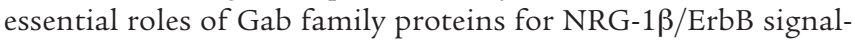
ing pathway in the heart. Gab1 and Gab2 were markedly tyro- 

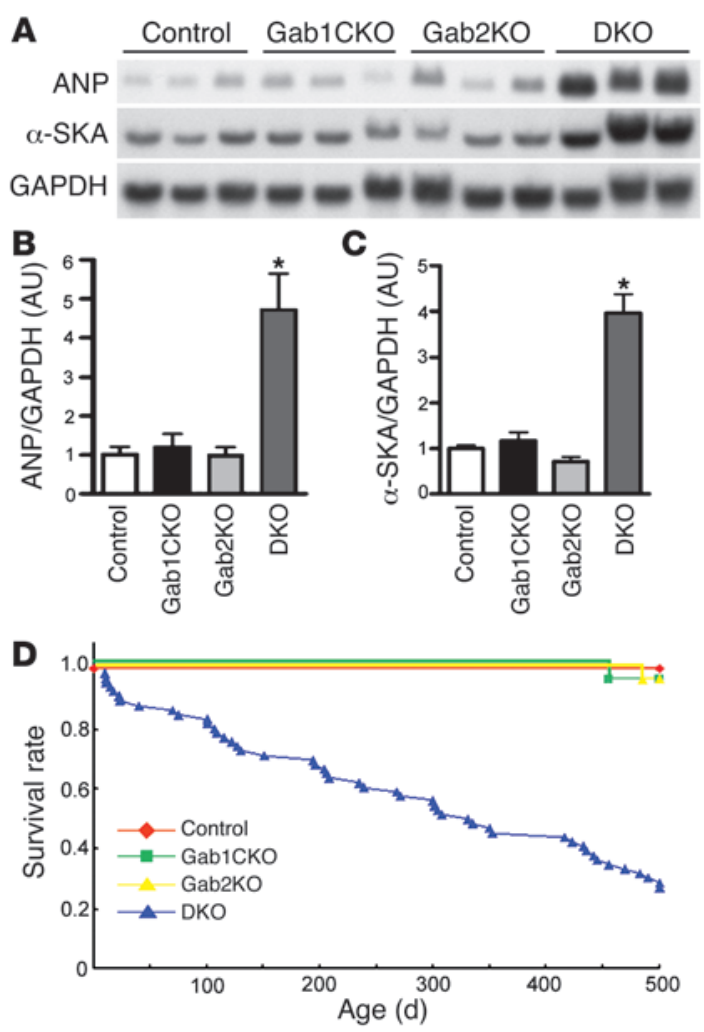

sine phosphorylated in the myocardium after stimulation with NRG-1 $\beta$ among various growth factors and cytokines. Tyrosinephosphorylated Gab1 and Gab2 subsequently associated with SHP2 and p85, resulting in strong activation of both ERK and AKT in the myocardium. NRG-1 $\beta$-dependent activation of ERK and AKT was almost completely abrogated in the DKO mouse hearts. In agreement with NRG-1 $\beta$-dependent downstream signaling defects, DKO mice displayed DCM-like phenotypes and EFE with aging. Interestingly, DKO mouse hearts also displayed abnormally dilated vessels with the loss of VSMCs. To address the mechanism for the abnormality in endocardial/vascular endothelium in DKO mouse hearts, we performed DNA microarray analysis and found several vasculature-regulating gene transcripts, such as Ang1, upregulated by NRG-1 $\beta$ in control, but not in DKO, mouse hearts. Thus, Gab family proteins mediate NRG-1 $\beta$-dependent stabilization of endocardial/vascular endothelium through the paracrine system from cardiomyocytes in the heart.

Gab1 and Gab2 are specifically required for coordination of NRG-1 $\beta /$ ErbB-dependent signaling pathway in the myocardium. NRG-1 $\beta$ shed from endothelial cells activates ErbB2/ErbB4 heterodimer or ErbB4 homodimer on the cardiomyocytes $(16,17$, $21)$. Consistent with this notion, we found that NRG-1 $\beta$ induced prominent tyrosine phosphorylation of Gab1 and Gab2 in cardiomyocytes but not in noncardiomyocytes. In addition, the cardiomyocyte-specific, high-MW isoform of Gab1 was tyrosine phosphorylated after stimulation with NRG-1 $\beta$ but not with other agonists including HB-EGF and EGF. It has been reported that HB-EGF-deficient mice develop heart failure $(25,26)$. Given that HB-EGF induced a much stronger tyrosine phosphorylation of Gab1 and Gab2 in noncardiomyocytes than in cardiomyocytes in our study and that valvular structures are developed from noncar-

\section{Figure 6}

DKO mice die of heart failure. (A) Northern blot analyses of the hearts from control, Gab1CKO, Gab2KO, and DKO mice ( $n=3$ for each group) at 12-14 weeks of age showed the increased expression of mRNAs for ANP and $\alpha-S K A$ in DKO mice. GAPDH mRNA was also measured for sample loading control. (B and $\mathbf{C}$ ) The relative levels of ANP and $\alpha$-SKA mRNA (normalized to GAPDH mRNA levels) were quantified from 3 mouse hearts in each group. ( ${ }^{\star} P<0.01$ compared with all other groups.) (D) Kaplan-Meier curves showing survival rate in control mice $(n=30)$, Gab1CKO mice $(n=30)$, Gab2KO mice $(n=30)$, and DKO mice $(n=66)$ mice by 500 days. The number of dead DKO mice was $48(72.7 \%) ; P<0.001$ for DKO versus control, Gab1CKO, and Gab2KO mice by log-rank test.

diomyocytes (19), the heart failure observed in HB-EGF-deficient mice might have resulted from abnormal signaling in the development of the valvular apparatus. Therefore, the cardiac phenotypes observed in DKO mice were mainly ascribable to the defects of the NRG-1 $\beta /$ ErbB signaling pathway in the myocardium. Consistent with this, similar DCM-like phenotypes are found in cardiac-specific ErbB2- and ErbB4-deficient mice (29-31).

NRG-1 $\beta$ activates both ERK and PI3K/AKT pathways in cardiomyocytes in vitro, both of which have been implicated in modulation of cell survival and protein synthesis $(21,43)$. NRG-1 $\beta$ actually induced strong activation of ERK and AKT in the hearts of control, but not DKO, mice. This finding provides what we believe to be the first in vivo evidence that Gab1 and Gab2 are required for transmission of the NRG-1 $\beta /$ ErbB signal to downstream signaling pathways, ERK and AKT. DKO mice progressively developed DCM phenotypes, demonstrating clearly that Gab1 and Gab2 were essential for maintenance of myocardial function through transmission of NRG-1 $\beta$ /ErbB signaling pathway (Figure 9).

DKO mice also exhibited abnormal deposition of elastic fibers and collagen specifically in the endocardium, reminiscent of the pathological features observed in primary EFE. Clinically, primary EFE is found mainly in infants, children, and adolescents and is frequently accompanied by contractile deterioration similar to DCM. Although there have been some reports suggesting the heritable causality of primary $\operatorname{EFE}(37,38)$, the precise pathogenetic mechanisms have not been elucidated to date. These DKO mice may provide the first mouse model of EFE. Further genetic analysis of cardiac-specific isoform of Gab1 will certainly contribute to our understanding of the pathogenesis of EFE.

DKO mouse hearts also displayed abnormal vasculatures as well as EFE. Microarray analysis enabled us to identify several transcripts that were upregulated by NRG-1 $\beta$ in the control hearts but not in DKO hearts. Among these transcripts selected in the cluster analysis, TSP1, EphA4, and Ang1 have been reported to be involved in the intercellular-dependent vascular regulation (40, $44,45)$. Intriguingly, NRG-1 $\beta /$ ErbB2/ErbB4 signaling, Ang1/Tie2 signaling, VEGF/VEGFR2 signaling, and serotonin-mediated $\left(5-\mathrm{HT}_{2 \mathrm{~B}}-\right.$ mediated) signaling are required for the proper maturation of endocardium $(16,17,40,46,47)$. Moreover, Ang1- or Tie2deficient mice exhibit embryonic lethality accompanied by abnormally dilated vessels as well as defects in the endocardium $(40,42$, 48). Furthermore, we demonstrated for the first time that postnatal cardiomyocytes are important Ang1-producing cells, whereas Ang1 has been believed to be mainly secreted from vascular mural cells such as pericytes and VSMCs $(40,41)$. Thus, we could pro- 

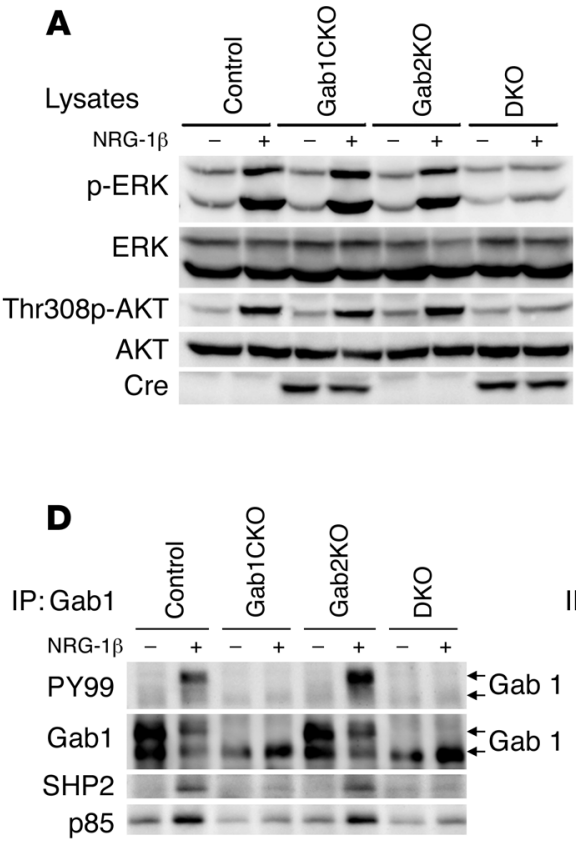

B
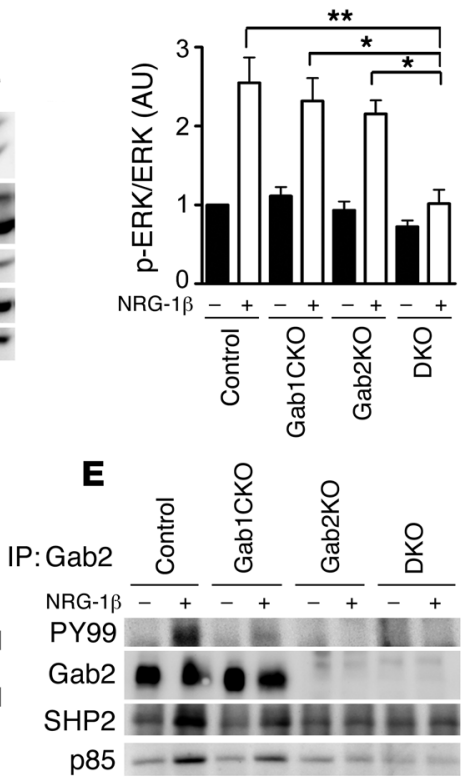

C
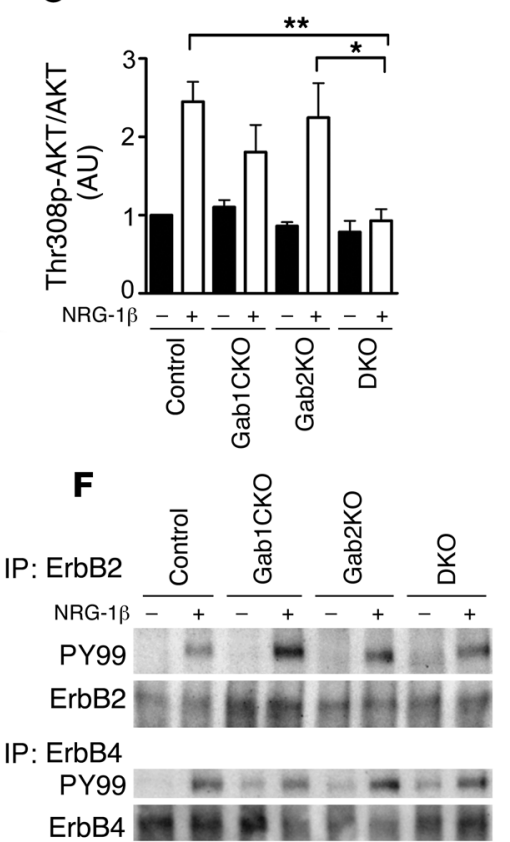

\section{Figure 7}

Gab1 and Gab2 are required for NRG-1 $\beta$-dependent ERK and AKT activation in the heart. (A) NRG-1 $\beta$-induced activation of ERK and AKT in the hearts from the indicated mice was assessed using phospho-specific Abs. Activation of ERK and AKT was exclusively attenuated in DKO hearts compared with the other 3 groups. Representative blots of 4 experiments are shown. (B) Phosphorylation of ERK was quantified against total ERK $(n=4)$. (C) Phosphorylation of AKT was quantified against total AKT $(n=4) .{ }^{*} P<0.05$, ${ }^{*} P<0.01$ for the indicated groups. Tyrosine phosphorylation of Gab1 (D) and Gab2 (E) and their association with SHP2 and p85 in hearts from the 4 groups of mice after injection with NRG-1 $\beta$ was examined as in Figure 1, A and B. Arrows in D denote the 2 isoforms of Gab1. (F) Tyrosine phosphorylation of ErbB2 (upper panels) and ErbB4 (lower panels) in hearts from the 4 groups were assessed at 5 minutes after NRG-1 $\beta$ injection. Tyrosine phosphorylation of ErbB receptors in the murine hearts upon NRG-1 $\beta$ stimulation was examined by IP with anti-ErbB2 or anti-ErbB4 Ab, followed by IB with the Abs indicated at the left.

pose that the defective expression of Ang1 might be involved in the pathogenesis of EFE and abnormal vessels in DKO hearts, though we cannot exclude the possibility that other vasculatureregulating genes, such as TSP1 and EphA4, play important roles in endocardial maintenance. Cardiac-specific gene ablation of Ang1 would be helpful to understand its importance in cardiomyocyteendothelial cell interactions.

So far, it has been well established that NRG-1 functions as a cytoprotective growth factor in cardiomyocytes $(17,21,43)$. Here, our findings propose a novel function of NRG-1; NRG-1 regulates vascular homeostasis through the paracrine expression of endothelium stabilization factors, such as Ang1, via Gab family proteins. Importantly, accumulating evidence has revealed that normal endothelial function is required for the maintenance of myocardial function (16). Collectively, Gab1 and Gab2 in the myocardium are essential for both maintenance of myocardial function and stabilization of capillary or endocardial endothelium through transmission of NRG-1 $\beta /$ ErbB signaling (Figure 9).

\section{Methods}

Materials. Anti-phospho-p44/p42 ERK (Thr202/Tyr204), anti-phosphoAKT (Thr308), and anti-AKT Abs were purchased from Cell Signaling Technology. The use of anti-Gab1 and anti-Gab2 serums in IP was described previously $(13,49)$. The Abs against Gab1, Gab2, and p85 used in IB analysis were from Millipore; Abs against antibody recognizing phospho-tyro- sine (PY99), ERK1, ERK2, and SHP2 were from Santa Cruz Biotechnology Inc.; $\mathrm{Abs}$ against $\mathrm{vWF}$ and $\alpha$-SMA were from Dako; the $\mathrm{Ab}$ against $\mathrm{CD} 31$ was from BD Biosciences - Pharmingen; and the Ab against Cre was from EMD Biosciences. Collagenase, Percoll, recombinant NRG-1 $\beta$ (NRG-1 $\beta$ EGF domain; sold as heregulin- $\beta 1$ ), HGF, and PDGF-BB were from SigmaAldrich. HB-EGF and EGF were from R\&D Systems. FGF2 was from EMD Biosciences. LIF was from Millipore. IGF-1 and erythropoietin were kindly provided by Astellas Pharma and Chugai Pharmaceutical Co., respectively.

Cell cultures. Primary cultures of neonatal rat cardiomyocytes were prepared from ventricles of 1- to 2-day-old Wistar rats (Kiwa Jikken Dobutsu) on Percoll gradient as described previously (32). Briefly, ventricles were isolated from neonatal rats and treated with trypsin and collagenase for 30 minutes at $37^{\circ} \mathrm{C}$. Isolated cells were suspended in $58.5 \%$ Percoll in HBSS $(20 \mathrm{mM}$ HEPES, $116 \mathrm{mM} \mathrm{NaCl}, 12.5 \mathrm{mM} \mathrm{NaH}_{2} \mathrm{PO}_{4}, 5.6 \mathrm{mM}$ glucose, $5.4 \mathrm{mM} \mathrm{KCl}$, $0.8 \mathrm{mM} \mathrm{MgSO}_{4} ; \mathrm{pH} 7.35$ ) and added to the discontinuous gradient consisting of $40.5 \%$ and $58.5 \%$ Percoll in HBSS. After centrifugation at $1,400 \mathrm{~g}$ for 30 minutes at $15^{\circ} \mathrm{C}$, the cardiomyocytes were collected from the interface of the discontinuous Percoll gradient and further enriched by preplating for 60 minutes on noncoated dishes. Unattached cells were cultured as cardiomyocytes in M-199 (Invitrogen) with 10\% FBS. Attached cells were cultured as noncardiomyocytes in DMEM with 10\% FBS. Immunocytochemical examination with anti-sarcomeric $\alpha$-actinin Ab (Sigma-Aldrich) revealed that more than $95 \%$ cultured cells in the cardiomyocyte fraction were sarcomeric $\alpha$-actinin-positive cardiomyocytes (data not shown). The population of noncardiomyocytes is described in the supplemental information. 
A

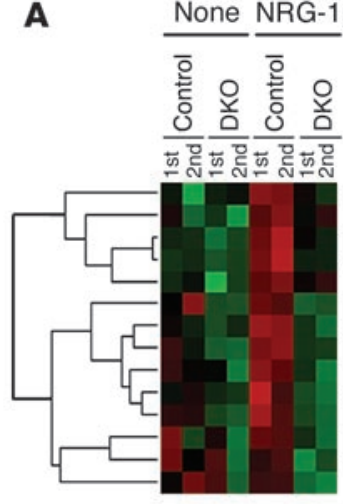

\section{Identification} Affymetrix Genbank $\begin{array}{ll}1421811 \text { at } & \mathrm{AV} 026492 \\ 1416125 & \text { at } \\ \text { BC } 015260\end{array}$ 1416125_at BC015260 1431213_a_at BG297038 1431214_at BG297038 1452426 x at BC004065 1418148 at NM_021304 1416204_at NM_01027 1434672_at BE 824968 1429196_at BB431654 1429021_at AK013481 1421488 at NM 013862

1448194_a at NM 023123 1447830_s_at BB 034265 log-fold induction rate

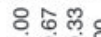
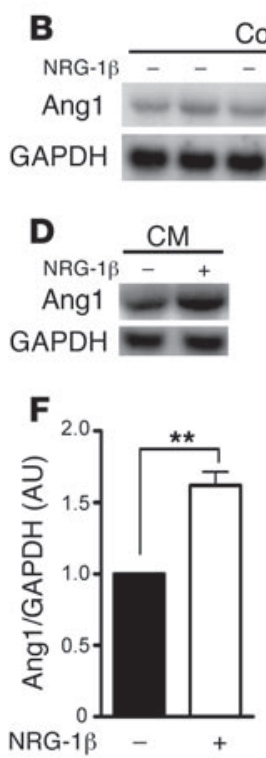

TSP1

FK506 binding protein 5

RIKEN cDNA $1300007 \mathrm{C} 21$

Similar to retrovirus-related POL polyprotein Zinc finger protein 236

Abhydrolase domain containing 1

Glycerol-3-phosphate dehydrogenase 1
G protein-coupled receptor 22

RIKEN cDNA 580411009 gene

EphA4

RAB GTPase activating protein1-like

$\mathrm{H} 19$ fetal liver mRNA

Regulator of $\mathrm{G}$ protein signaling 2

\section{(-)}

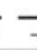

DKO

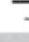
$-+++$

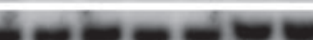

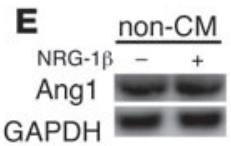

G

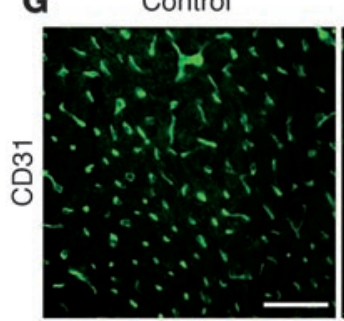

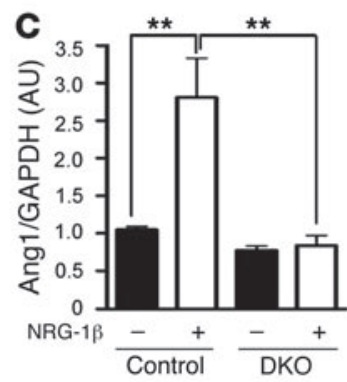

DKO

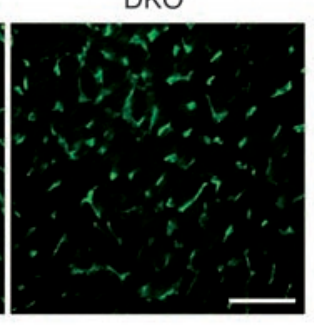

H

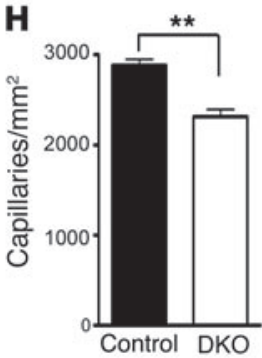

\section{Figure 8}

Gab1 and Gab2 are required for the NRG-1 $\beta$-induced Ang1 upregulation and endothelial stabilization in the heart. (A) RNAs from the ventricles of control and DKO mice ( $n=3$ per group) were prepared 8 hours after injection with NRG-1 $\beta$ or vehicle. This preparation was performed twice. We used pooled RNAs from 3 mice and performed Affymetrix DNA microarrays independently 2 times (indicated as 1 st and 2 nd). Cluster analysis was performed of upregulated (red) and downregulated (green) genes in NRG-1 $\beta$-treated control and DKO mice. Color intensity is relative to the median (black). (B) Northern blot analysis demonstrated the upregulation of Ang1 mRNA in the hearts of control mice but not in those of DKO mice following injection with NRG-1 $\beta$ ( $n=3$ per group). GAPDH mRNA was checked for gel loading. (C) Quantitative analysis of Ang1 mRNA (normalized to GAPDH mRNA) ( $n=3$ per group; ${ }^{* \star} P<0.01$ between the indicated groups). (D and E) Ang1 expression was upregulated in cardiomyocytes but not in noncardiomyocytes after stimulation with NRG-1 $\beta$. (F) Quantitative analysis of Ang1 mRNA normalized to GAPDH mRNA $\left(n=4 ;{ }^{* *} P<0.01\right)$. (G) Cryosections of the hearts from control and DKO mice were immunostained with anti-CD31 Ab. Representative results are shown. Scale bars: $50 \mu \mathrm{m}$. (H) Capillary densities were counted from the number of capillaries per square millimeter. ${ }^{\star \star} P<0.01$.
Biochemical analyses. Mice (body weight, approximately $20 \mathrm{~g}$ ) were injected with following agonists: LIF $\left(1 \times 10^{4} \mathrm{U}\right)$, NRG-1 $\beta(5 \mu \mathrm{g})$, HB-EGF $(5 \mu \mathrm{g})$, EGF $(5 \mu \mathrm{g})$, FGF2 $(2 \mu \mathrm{g})$, IGF-1 $(5 \mu \mathrm{g})$, HGF $(2 \mu \mathrm{g})$, PDGF-BB $(5 \mu \mathrm{g})$, and erythropoietin $(600 \mathrm{IU})$. Briefly, mice anesthetized by avertin were injected i.v. via the inferior vena cava with various growth factors and cytokines dissolved in $100 \mu \mathrm{l}$ of normal saline. The hearts were isolated 5 minutes after injection and washed with ice-cold $4^{\circ} \mathrm{C}$ PBS. After removing both atria, the ventricles were snap-frozen in liquid nitrogen. The ventricles were homogenized in lysis buffer containing $50 \mathrm{mM}$ HEPES, $100 \mathrm{mM}$ sodium fluoride, $2 \mathrm{mM}$ sodium orthovanadate, $4 \mathrm{mM}$ EDTA, $1 \%$ Tween- $20,0.1 \%$ SDS, and a protease inhibitor cocktail Complete (Roche Applied Science) using a polytron homogenizer as described previously (50). The lysates were cleared by centrifugation at $17,000 \mathrm{~g}$ for 30 minutes. Protein concentration was measured with BCA protein assay kit (Pierce). Lysates of cultured cells were prepared essentially as described previously (13). After stimulation, cells were immediately lysed in ice-cold lysis buffer (20 mM Tris- $\mathrm{HCl}$ ( $\mathrm{pH} 7.4), 150$ $\mathrm{mM} \mathrm{NaCl}, 1 \% \mathrm{NP}-40,1 \mathrm{mM}$ sodium vanadate, $1 \mathrm{mM}$ dithiothreitol, and a protease inhibitor cocktail Complete). The cleared lysates were subjected to IP and IB following standard procedures as described previously (13).

Creation of a conditional Gab1 mutant allele. Genomic DNA fragments of Gab1 were isolated from a $\lambda$-FixII 129/Sv mouse strain genomic library, and a targeting construct was engineered using a triple-loxP system (provided by W. Reith, Department of Pathology and Immunology, University of Geneva Medical School, Geneva, Switzerland). We flanked exon 2, which encodes part of pleckstrin homology domain with 2 loxP sites (which codes for amino acids 25-123). The linearized construct DNA was introduced into R1 embryonic stem cells by electroporation. G418-resistant cell colonies were screened for homologous recombination by PCR, as reconfirmed by Southern blot analysis. Embryonic stem cell clones with a loxP-floxed exon 2 $\left(G a b 1^{f l o x}\right)$ without the TK-neo cassette were obtained by transient transfection with a pCre-Pac (provided by T. Yagi, Osaka University) (51). The engineered embryonic stem cells were injected into C57BL/6J blastocysts.

Generation of Gab1CKO and DKO mice. Gab1flox allele was generated in 129/Sv-C57BL/6J mixed background. The transgenic mice expressing Cre recombinase under the control of $\alpha$-MHC promoter in C57BL/6J background ( $\alpha$-MHC-Cre mice) were generated as previously reported (35). Gab 1floxfflox mice were crossed with $\alpha$-MHC-Cre mice to generate Gab1CKO mice $\left(\mathrm{Gab} 1^{\text {flox } / f l o x} \mathrm{Gab2} 2^{+/+} \alpha-M H C-C r e(+)\right)$. We had also previously created Gab2KO mice and reported that these mice are viable and display defects in mast cells $(9,11)$. We further crossed Gab1CKO mice with Gab2KO mice

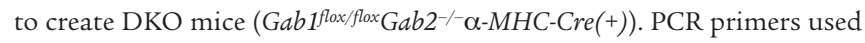
for mouse genotyping are listed in the supplemental information. All mice 
Endocardial or capillary endothelium

Endothelial stabilization

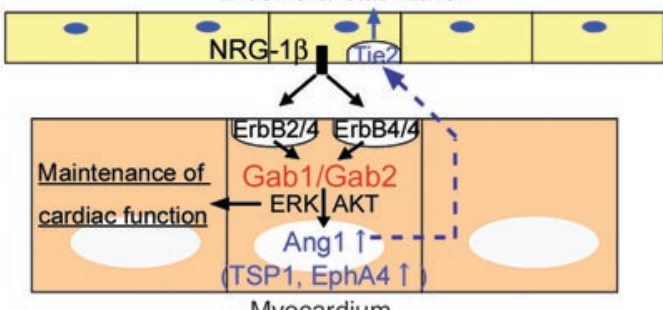

Myocardium

\section{Figure 9}

Schematic illustration of the roles of Gab family proteins in the myocardium. NRG-1 $\beta$ shed from the endocardial or capillary endothelium in the heart activates ErbB receptors on the myocardium, resulting in tyrosine phosphorylation of Gab family proteins and subsequent activation of ERK and AKT. NRG-1//ErbB-Gab1/Gab2 signaling in the myocardium is directly required for postnatal maintenance of myocardial function. In addition, NRG-1//ErbB-Gab1/Gab2 signaling indirectly contributes to postnatal stabilization of capillary or endocardial endothelium, possibly through Ang1 upregulation (dotted line).

were maintained on a 129/Sv-C57BL/6J mixed background. We housed all animals in a virus-free facility on a 12-hour light/12-hour dark cycle and fed them a standard mouse food. Animal experiments were approved by the National Cardiovascular Center Research Committee and were performed according to the institutional guidelines.

Histological analyses. Hearts from mice at indicated ages (see Results and Figure 3 legend) were fixed with 10\% neutralized formalin, embedded in paraffin, and sectioned at $4 \mu \mathrm{m}$ thickness. Masson trichrome and elastica van Gieson staining were performed on serial sections. To examine the capillary density, hearts of 4- to 6-week-old mice were fixed with $4 \%$ paraformaldehyde, cryoprotected with $20 \%$ sucrose, and frozen in OCT compound (Sakura). Cryosections $(10 \mu \mathrm{m})$ were stained with rat monoclonal anti-CD31 Ab and FITC-conjugated secondary Ab. Paraffin sections of hearts from 4- to 6-week-old mice were immunostained with anti-vWF or anti- $\alpha$-SMA Ab from the EnVision+ Kit (Dako) according to the manufacturer's instructions.

Physiological analyses. For echocardiography, male mice at the indicated ages were anesthetized with $2.5 \%$ avertin (Wako; $15 \mu \mathrm{l} / \mathrm{g}$ body weight). Echocardiography was performed using a Hewlett Packard Sonos 5500 Echocardiography System and a $15-\mathrm{MHz}$ linear transducer. Ventricular dimensions were measured on $\mathrm{M}$-mode images at least 3 times for each group of mice. For hemodynamic measurements, 12-week-old male mice were anesthetized with i.p. injection of urethane $(750 \mathrm{mg} / \mathrm{kg})$ and $\alpha$-chloralose $(50 \mathrm{mg} / \mathrm{kg})$ dissolved in normal saline (52). The right common carotid artery was exposed via the midline incision. To allow the use of a physiologic closed-chest preparation, the LV was catheterized retrogradely from the carotid artery using a high-fidelity pressure transducer catheter (1.4 French; Millar Instruments) (53). The LV pressure was digitized, stored on the hard disk of a dedicated laboratory computer system, and analyzed with custom software.

Northern blot analysis. The probes for ANP, $\alpha$-SKA, and GAPDH were kindly donated by K.R. Chien (Massachusetts General Hospital, Boston, Massachusetts, USA). Total RNA was prepared by TRIzol reagent (Invitrogen) according to the manufacturer's instructions. RNAs from the ventricles of mouse hearts and cultured cardiomyocytes were subjected to Northern blot analyses against a panel of cDNA probes indicated in Results and the legends for Figures 6 and 8. Hybridization was performed using Quikhyb (Stratagene). Quantitative data were collected after normalizing the results to GAPDH.

Gene expression profiling using Affymetrix DNA microarrays. Gene expression in cardiac ventricular tissue was analyzed by Affymetrix microarray hybridization. Control and DKO male mice at 6 weeks of age were injected with $5 \mu \mathrm{g}$ of NRG- $1 \beta$ or vehicle (normal saline) via tail vein. The ventricles were isolated at 8 hours after injection and subjected to extraction of total RNA using TRIzOL reagent. We pooled total RNA from 3 mouse ventricles for each group and subjected to the microarray hybridization as described previously (54). Preparation of cRNA and hybridization of probe on arrays were performed according to the manufacturer's instructions (Affymetrix). Each array experiment was performed in duplicate. FileMaker Pro 8.0 software was used to analyze genes that demonstrated identical patterns in 2 independent microarray experiments. Data were analyzed according to the minimum information about a microarray experiment (MIAME) rule. Annotation of the probe numbers and targeted sequences are shown on the Affymetrix website (https://www.affymetrix.com/site/login/login.affx).

Statistics. All data are expressed as mean \pm SEM. Differences among multiple groups were compared by 1-way ANOVA followed by a post-hoc comparison using Scheffe's method. The 2-tailed Student's $t$ test was used to analyze differences between 2 groups. $P<0.05$ was considered statistically significant. Survival curves were generated using Kaplan-Meier method, and significance was evaluated using the log-rank test.

\section{Acknowledgments}

This work was supported in part by grants from the Ministry of Education, Culture, Sports, Science, and Technology of Japan (to Y. Nakaoka and N. Mochizuki); the Ministry of Health, Labour, and Welfare of Japan (to N. Mochizuki); the Program for the Promotion of Fundamental Studies in Health Sciences of the National Institute of Biomedical Innovation (to T. Minami, T. Kodama, and N. Mochizuki); the Japan Heart Foundation (Novartis Grant for Research Award on Molecular and Cellular Cardiology, to Y. Nakaoka); the Mitsubishi Pharma Research Foundation (to Y. Nakaoka); and the Uehara Memorial Foundation (to Y. Nakaoka). We thank Y. Matsuura, M. Yoshida, M. Miyabayashi, M. Maeoka, Y. Ohba, M. Sato, K. Sako, and K. Shioya for their technical assistance; S. Higashiyama, O. Nakagawa, and O. Ohara for helpful comments; K. Komamura for advice on echocardiography; and W.W. Hall and K. Yamauchi-Takihara for critical reading of the manuscript.

Received for publication October 16, 2006, and accepted in revised form April 10, 2007.

Address correspondence to: Naoki Mochizuki, Department of Structural Analysis, National Cardiovascular Center Research Institute, 5-7-1 Fujishirodai, Suita, Osaka 565-8565, Japan. Phone: 81-6-6833-5012, ext. 2508; Fax: 81-6-6835-5461; E-mail: nmochizu@ri.ncvc.go.jp. Or to: Yoshikazu Nakaoka: Department of Cardiovascular Medicine, Osaka University Graduate School of Medicine, 2-2, Yamada-oka, Suita, Osaka 565-0871, Japan. Phone: 81-6-6879-3835; Fax: 81-6-6879-3839; E-mail: ynakaoka@ imed3.med.osaka-u.ac.jp.

Y. Nakaoka's present address is: Department of Cardiovascular Medicine, Osaka University Graduate School of Medicine, Osaka, Japan.

Hisao Hirota is deceased. 
1. Seidman, J.G., and Seidman, C. 2001. The genetic basis for cardiomyopathy: from mutation identification to mechanistic paradigms. Cell. 104:557-567.

2. Chien, K.R. 1999. Stress pathways and heart failure. Cell. 98:555-558.

3. Schlessinger, J., and Lemmon, M.A. 2003. SH2 and PTB domains in tyrosine kinase signaling. Sci. STKE. 2003:RE12.

4. Gu, H., and Neel, B.G. 2003. The "Gab" in signal transduction. Trends Cell Biol. 13:122-130.

5. Nishida, K., and Hirano, T. 2003. The role of Gab family scaffolding adapter proteins in the signal transduction of cytokine and growth factor receptors. Cancer Sci. 94:1029-1033.

6. Itoh, M., et al. 2000. Role of Gab1 in heart, placenta, and skin development and growth factor- and cytokine-induced extracellular signal-regulated kinase mitogen-activated protein kinase activation. Mol. Cell. Biol. 20:3695-3704.

7. Sachs, M., et al. 2000. Essential role of Gab1 for signaling by the c-Met receptor in vivo. J. Cell Biol. 150:1375-1384.

8. Gu, H., et al. 2001. Essential role for Gab2 in the allergic response. Nature. 412:186-190.

9. Nishida, K., et al. 2002. Requirement of Gab2 for mast cell development and KitL/c-Kit signaling. Blood. 99:1866-1869.

10. Wada, T., et al. 2005. The molecular scaffold Gab2 is a crucial component of RANK signaling and osteoclastogenesis. Nat. Med. 11:394-399.

11. Nishida, K., et al. 2005. FcepsilonRI-mediated mast cell degranulation requires calcium-independent microtubule-dependent translocation of granules to the plasma membrane. J. Cell Biol. 170:115-126.

12. Seiffert, M., et al. 2003. Gab3-deficient mice exhibit normal development and hematopoiesis and are immunocompetent. Mol. Cell. Biol. 23:2415-2424.

13. Nakaoka, Y., et al. 2003. Activation of gp130 transduces hypertrophic signal through interaction of scaffolding/docking protein Gab1 with tyrosine phosphatase SHP2 in cardiomyocytes. Circ. Res. 93:221-229.

14. Bentires-Alj, M., et al. 2006. A role for the scaffolding adapter GAB2 in breast cancer. Nat. Med. 12:114-121.

15. Yamasaki, S., et al. 2003. Gab1 is required for EGF receptor signaling and the transformation by activated ErbB2. Oncogene. 22:1546-1556.

16. Brutsaert, D.L. 2003. Cardiac endothelial-myocardial signaling: its role in cardiac growth, contractile performance, and rhythmicity. Physiol. Rev. 83:59-115.

17. Garratt, A.N., Ozcelik, C., and Birchmeier, C. 2003. ErbB2 pathways in heart and neural diseases. Trends Cardiovasc. Med. 13:80-86.

18. Falls, D.L. 2003. Neuregulins: functions, forms, and signaling strategies. Exp. Cell Res. 284:14-30.

19. Iwamoto, R., and Mekada, E. 2006. ErbB and HBEGF signaling in heart development and function. Cell Struct. Funct. 31:1-14.

20. Lemmens, K., Segers, V.F., Demolder, M., and De Keulenaer, G.W. 2006. Role of neuregulin-1/ErbB2 signaling in endothelium-cardiomyocyte crosstalk. J. Biol. Chem. 281:19469-19477.
21. Zhao, Y.Y., et al. 1998. Neuregulins promote survival and growth of cardiac myocytes. Persistence of ErbB2 and ErbB4 expression in neonatal and adult ventricular myocytes. J. Biol. Chem. 273:10261-10269.

22. Gassmann, M., et al. 1995. Aberrant neural and cardiac development in mice lacking the ErbB4 neuregulin receptor. Nature. 378:390-394.

23. Lee, K.F., et al. 1995. Requirement for neuregulin receptor erbB2 in neural and cardiac development. Nature. 378:394-398.

24. Meyer, D., and Birchmeier, C. 1995. Multiple essential functions of neuregulin in development. Nature. 378:386-390.

25. Iwamoto, R., et al. 2003. Heparin-binding EGFlike growth factor and ErbB signaling is essential for heart function. Proc. Natl. Acad. Sci. U. S. A. 100:3221-3226.

26. Jackson, L.F., et al. 2003. Defective valvulogenesis in HB-EGF and TACE-null mice is associated with aberrant BMP signaling. EMBO J. 22:2704-2716.

27. Slamon, D.J., et al. 2001. Use of chemotherapy plus a monoclonal antibody against HER2 for metastatic breast cancer that overexpresses HER2. N. Engl.J. Med. 344:783-792.

28. Suter, T.M., Cook-Bruns, N., and Barton, C. 2004. Cardiotoxicity associated with trastuzumab (Herceptin) therapy in the treatment of metastatic breast cancer. Breast. 13:173-183.

29. Crone, S.A., et al. 2002. ErbB2 is essential in the prevention of dilated cardiomyopathy. Nat. Med. 8:459-465.

30. Garcia-Rivello, H., et al. 2005. Dilated cardiomyopathy in Erb-b4-deficient ventricular muscle. Am. J. Physiol. Heart Circ. Physiol. 289:H1153-H1160.

31. Ozcelik, C., et al. 2002. Conditional mutation of the ErbB2 (HER2) receptor in cardiomyocytes leads to dilated cardiomyopathy. Proc. Natl. Acad. Sci. U. S. A. 99:8880-8885.

32. Nakagawa, O., et al. 1995. Rapid transcriptional activation and early mRNA turnover of brain natriuretic peptide in cardiocyte hypertrophy. Evidence for brain natriuretic peptide as an "emergency" cardiac hormone against ventricular overload. J. Clin. Invest. 96:1280-1287.

33. Cote, G.M., Miller, T.A., Lebrasseur, N.K., Kuramochi, Y., and Sawyer, D.B. 2005. Neuregulin-1alpha and beta isoform expression in cardiac microvascular endothelial cells and function in cardiac myocytes in vitro. Exp. Cell Res. 311:135-146.

34. Agah, R., et al. 1997. Gene recombination in postmitotic cells. Targeted expression of Cre recombinase provokes cardiac-restricted, site-specific rearrangement in adult ventricular muscle in vivo. J. Clin. Invest. 100:169-179.

35. Yamaguchi, O., et al. 2004. Cardiac-specific disruption of the c-raf-1 gene induces cardiac dysfunction and apoptosis. J. Clin. Invest. 114:937-943. doi:10.1172/JCI200420317.

36. Meng, S., Chen, Z., Munoz-Antonia, T., and Wu, J. 2005. Participation of both Gab1 and Gab2 in the activation of the ERK/MAPK pathway by epidermal growth factor. Biochem. J. 391:143-151.

37. Moss, A.J., and Adams, F.H. 1995. Moss' heart dis- ease in infants, children, and adolescents. Williams \& Wilkins. Baltimore, Maryland, USA. 1,085 pp.

38. Westwood, M., Harris, R., Burn, J.L., and Barson, A.J. 1975. Heredity in primary endocardial fibroelastosis. Br. Heart J. 37:1077-1084.

39. Xu, X., et al. 2005. ASF/SF2-regulated CaMKIIdelta alternative splicing temporally reprograms excitation-contraction coupling in cardiac muscle. Cell. 120:59-72.

40. Brindle, N.P., Saharinen, P., and Alitalo, K. 2006. Signaling and functions of angiopoietin- 1 in vascular protection. Circ. Res. 98:1014-1023.

41. Davis, S., et al. 1996. Isolation of angiopoietin-1, a ligand for the TIE2 receptor, by secretion-trap expression cloning. Cell. 87:1161-1169.

42. Suri, C., et al. 1996. Requisite role of angiopoietin-1, a ligand for the TIE2 receptor, during embryonic angiogenesis. Cell. 87:1171-1180.

43. Baliga, R.R., et al. 1999. NRG-1-induced cardiomyocyte hypertrophy. Role of PI-3-kinase, p70(S6K), and MEK-MAPK-RSK. Am. J. Physiol. 277:H2026-H2037.

44. Lawler,J. 2000. The functions of thrombospondin-1 and-2. Curr. Opin. Cell Biol. 12:634-640.

45. Ogita, H., et al. 2003. EphA4-mediated Rho activation via Vsm-RhoGEF expressed specifically in vascular smooth muscle cells. Circ. Res. 93:23-31.

46. Liao, W., et al. 1997. The zebrafish gene cloche acts upstream of a flk-1 homologue to regulate endothelial cell differentiation. Development. 124:381-389.

47. Nebigil, C.G., et al. 2000. Serotonin 2B receptor is required for heart development. Proc. Natl. Acad. Sci. U. S. A. 97:9508-9513.

48. Sato, T.N., et al. 1995. Distinct roles of the receptor tyrosine kinases Tie-1 and Tie-2 in blood vessel formation. Nature. 376:70-74.

49. Nishida, K., et al. 1999. Gab-family adapter proteins act downstream of cytokine and growth factor receptors and $\mathrm{T}$ - and $\mathrm{B}$-cell antigen receptors. Blood. 93:1809-1816.

50. Osugi, T., et al. 2002. Cardiac-specific activation of signal transducer and activator of transcription 3 promotes vascular formation in the heart. J. Biol. Chem. 277:6676-6681.

51. Taniguchi, M., et al. 1998. Efficient production of Cre-mediated site-directed recombinants through the utilization of the puromycin resistance gene, pac: a transient gene-integration marker for ES cells. Nucleic Acids Res. 26:679-680.

52. Georgakopoulos, D., et al. 1998. In vivo murine left ventricular pressure-volume relations by miniaturized conductance micromanometry. Am. J. Physiol. 274:H1416-H1422.

53. Nishio, R., Sasayama, S., and Matsumori, A. 2002. Left ventricular pressure-volume relationship in a murine model of congestive heart failure due to acute viral myocarditis. J. Am. Coll. Cardiol. 40:1506-1514.

54. Minami, T., Miura, M., Aird, W.C., and Kodama, T. 2006. Thrombin-induced autoinhibitory factor, Down syndrome critical region-1, attenuates NFAT-dependent vascular cell adhesion molecule-1 expression and inflammation in the endothelium. J. Biol. Chem. 281:20503-20520. 